\title{
Noise-induced neurophysiological alterations in the rat medial geniculate body and thalamocortical desynchronization by deep brain stimulation
}

Citation for published version (APA):

van Zwieten, G., Roberts, M. J., Schaper, F. L. V. W., Smit, J. V., Temel, Y., \& Janssen, M. L. F. (2021). Noise-induced neurophysiological alterations in the rat medial geniculate body and thalamocortical desynchronization by deep brain stimulation. Journal of Neurophysiology, 125(2), 661-671. https://doi.org/10.1152/jn.00752.2019

Document status and date:

Published: 01/02/2021

DOI:

10.1152/jn.00752.2019

Document Version:

Publisher's PDF, also known as Version of record

\section{Document license:}

Taverne

Please check the document version of this publication:

- A submitted manuscript is the version of the article upon submission and before peer-review. There can be important differences between the submitted version and the official published version of record.

People interested in the research are advised to contact the author for the final version of the publication, or visit the DOI to the publisher's website.

- The final author version and the galley proof are versions of the publication after peer review.

- The final published version features the final layout of the paper including the volume, issue and page numbers.

Link to publication

\footnotetext{
General rights rights.

- You may freely distribute the URL identifying the publication in the public portal. please follow below link for the End User Agreement:

www.umlib.nl/taverne-license

Take down policy

If you believe that this document breaches copyright please contact us at:

repository@maastrichtuniversity.nl

providing details and we will investigate your claim.
}

Copyright and moral rights for the publications made accessible in the public portal are retained by the authors and/or other copyright owners and it is a condition of accessing publications that users recognise and abide by the legal requirements associated with these

- Users may download and print one copy of any publication from the public portal for the purpose of private study or research.

- You may not further distribute the material or use it for any profit-making activity or commercial gain

If the publication is distributed under the terms of Article $25 \mathrm{fa}$ of the Dutch Copyright Act, indicated by the "Taverne" license above, 


\title{
Noise-induced neurophysiological alterations in the rat medial geniculate body and thalamocortical desynchronization by deep brain stimulation
}

\author{
Gusta van Zwieten, ${ }^{1,2}$ (1) Mark J. Roberts, ${ }^{3}$ Frédéric L. V. W Schaper, ${ }^{2,4}$ Jasper V. Smit, ${ }^{2,5}$ Yasin Temel, ${ }^{2,4}$ \\ and Marcus L. F. Janssen ${ }^{2,6}$ \\ ${ }^{1}$ Department of Ear Nose and Throat/Head and Neck Surgery, Maastricht University Medical Center, Maastricht, The \\ Netherlands; ' School of Mental Health and Neuroscience, Maastricht University Medical Center, Maastricht, The Netherlands; \\ ${ }^{3}$ Department of Cognitive Neuroscience, Faculty of Psychology and Neuroscience, Maastricht University, Maastricht, The \\ Netherlands; ${ }^{4}$ Department of Neurosurgery, Maastricht University Medical Center, Maastricht, The Netherlands; ${ }^{5}$ Department \\ of Ear Nose and Throat/Head and Neck Surgery, Zuyderland Hospital, Heerlen, The Netherlands; and ${ }^{6}$ Department of \\ Clinical Neurophysiology, Maastricht University Medical Center, Maastricht, The Netherlands
}

\begin{abstract}
The thalamic medial geniculate body (MGB) is uniquely positioned within the neural tinnitus networks. Deep brain stimulation (DBS) of the MGB has been proposed as a possible novel treatment for tinnitus, yet mechanisms remain elusive. The aim of this study was to characterize neurophysiologic hallmarks in the MGB after noise exposure and to assess the neurophysiological effects of electrical stimulation of the MGB. Fourteen male Sprague-Dawley rats were included. Nine subjects were unilaterally exposed to a 16-kHz octave-band noise at $115 \mathrm{~dB}$ for $90 \mathrm{~min}$, five received sham exposure. Single units were recorded from the contralateral MGB where spontaneous firing, coefficient of variation, response type, rate-level functions, and thresholds were determined. Local field potentials and electroencephalographical (EEG) recordings were performed before and after high-frequency DBS of the MGB. Thalamocortical synchronization and power were analyzed. In total, 214 single units were identified ( $n=145$ in noise-exposed group, $n=69$ in control group). After noise exposure, fast-responding neurons become less responsive or nonresponsive without change to their spontaneous rate, whereas sustained- and suppressed-type neurons exhibit enhanced spontaneous activity without change to their stimulus-driven activity. MGB DBS suppressed thalamocortical synchronization in the $\beta$ and $\gamma$ bands, supporting suppression of thalamocortical synchronization as an underlying mechanism of tinnitus suppression by high frequency DBS. These findings contribute to our understanding of the neurophysiologic consequences of noise exposure and the mechanism of potential DBS therapy for tinnitus.
\end{abstract}

NEW \& NOTEWORTHY Separate functional classes of MGB neurons might have distinct roles in tinnitus pathophysiology. After noise exposure, fast-responding neurons become less responsive or nonresponsive without change to their spontaneous firing, whereas sustained and suppressed neurons exhibit enhanced spontaneous activity without change to their stimulus-driven activity. Furthermore, results suggest desynchronization of thalamocortical $\beta$ and $\gamma$ oscillations as a mechanism of tinnitus suppression by MGB DBS.

auditory thalamus; deep brain stimulation; electrophysiology; medial geniculate body; tinnitus

\section{INTRODUCTION}

Acoustic overexposure is the most common cause of tinnitus (1). Patients suffering from tinnitus perceive a phantom sound. To date, the exact pathophysiological mechanisms involved in tinnitus are still poorly understood. According to the most accepted theory, tinnitus is generated in response to a reduction of input from the cochlea to the brain. Even though tinnitus often arises after hearing loss, persons with hearing loss do not always develop tinnitus. Therefore, it has been postulated that not only bottom-up deafferentation but also a deficient top-down noise-cancelling mechanism plays 
a role in tinnitus development $(2,3)$. Overall, a hyperactive state is found within tinnitus-related brain structures, characterized by increased spontaneous and bursting activity, tonotopic reorganization, and enhanced local and longrange neural synchrony (4-6).

The medial geniculate body (MGB) is part of the auditory thalamus and has a unique position in auditory pathophysiology (7). This structure acts as a central hub in purported tinnitus networks, as it connects to both auditory and limbic structures (3). It has been suggested that the MGB plays a significant role in not only the auditory but also the emotional and attentional aspects of tinnitus (8,9). MGB neurons integrate and shape neural representations of auditory information and control passage to the cerebral cortex (10). The ventral division of the MGB receives auditory input from the central inferior colliculus and mainly projects to the primary auditory cortex. The medial and dorsal MGB receives input from dorsal and external cortex of the inferior colliculus, which further projects to belt regions of the auditory cortex. Moreover, paralimbic structures connect via the thalamic reticular nucleus to the MGB, which acts as a thalamic gate and might function as a "noise-cancelling system." Two tinnitus models propose essential involvement of the MGB in tinnitus pathophysiology. First, it has been suggested that the inhibitory feedback loop (or thalamic gate) does not work sufficiently in tinnitus patients (3). Second, thalamocortical oscillations and increased coherence among highand low-frequency oscillations indicate presence of thalamocortical dysrhythmia in tinnitus and might be responsible for the phantom sound $(2,11)$.

Despite its central position in the auditory network and multiple pathophysiological hypotheses, the MGB has received little attention in neurophysiological studies. Although electrophysiological recordings in human MGB are unavailable, structural and functional abnormalities in the thalamus have been investigated in tinnitus patients using imaging studies (12). A high-resolution magnetic resonance imaging (MRI) study using voxel-based morphometry showed increased gray-matter volume at the auditory thalamus (13) relative to healthy participants, although these findings could not be replicated by others (14). In a functional MRI study, lateralized activation of MGB was found in unilateral tinnitus, whereas bilateral tinnitus was associated with bilateral activation pattern (15).

Thus far, only few MGB-related neurophysiological studies in the noise-exposed animal model have been reported. In one study, an increase in spontaneous activity, burst properties and sound-evoked activity of MGB neurons was found in awake rats with behavioral evidence of tinnitus (16). In another study, noise-exposed anesthetized tinnitus rats did not show increased spontaneous firing rates but showed a reduction in neurons with bursty firing (17). In an in vitro study on mice brain slices, the firing rate of MGB neurons was increased in some neurons and decreased in others after application of salicylate, which is known to induce temporary tinnitus (18). In one of our recent studies, MGB DBS was effective in suppressing tinnitus-like behavior in a noise-induced animal model (19). We, therefore, propose the MGB as a potential therapeutic target in deep brain stimulation (DBS) for tinnitus (20).
The aim of this study was to further characterize the neurophysiologic fingerprint of the MGB in the noise-exposed animal model and to assess the neurophysiological effects of electrical stimulation of the MGB. More insight in neuropathophysiological changes after noise exposure will contribute to further unravel physiological, but also pathological auditory processing such as in case of tinnitus. To this aim, single unit recordings from noise-exposed rodents and unexposed control animals were recorded. To explore the working mechanism of MGB DBS, spontaneous local field potentials (LFPs) and electroencephalographical (EEG) recordings were performed before and after application of high-frequency DBS in the MGB.

\section{MATERIAL AND METHODS}

\section{Subjects and Experimental Design}

Fourteen male Sprague-Dawley rats (Charles River, Sulzfeld, Germany) were included in this study, aged $7 \mathrm{wk}$ and weighing $\pm 250 \mathrm{~g}$ at the start of the experiment. Animals were housed in pairs with ad libitum access to food and water, and with a reversed day/night cycle. Experiments were conducted in the dark period of the day. The experimental protocol was approved by the Animal Experiments and Ethics Committee of Maastricht University. Subjects were divided in two groups: noise exposure $(n=9)$ and control $(n=5)$.

\section{Noise Exposure}

All subjects were anesthetized by use of ketamine $(10 \mathrm{mg} / \mathrm{kg}$ and for maintenance $60 \mathrm{mg} / \mathrm{kg} / \mathrm{h})$ and xylazine $(90 \mathrm{mg} / \mathrm{kg})$. Subjects in the noise-exposed group were unilaterally exposed to a $16 \mathrm{kHz}$ octave-band noise at $115 \mathrm{~dB}$ for 90 min (Ultrasonic Power Amplifier and Ultrasonic Dynamic Speaker Vifa; Avisoft Bioacoustics, Berlin, Germany), which was calibrated at speaker-level (Bruel \& Kjaer $2231 \mathrm{~dB}$ meter and 4191 microphone). A plug of clay protected the contralateral ear from noise.

\section{Gap Prepulse Inhibition of Acoustic Startles}

Behavioral testing of tinnitus was conducted using Gap Prepulse Inhibition of Acoustic Startle response paradigm [GPIAS, detailed description has been provided elsewhere (21)]. All subjects were tested in two conditions: baseline and 3 wk after noise or sham exposure. Briefly, rats were placed inside a cylinder with vertical aluminum bars and a polyethylene floor (diameter $17 \mathrm{~cm}$, height $40 \mathrm{~cm}$ ), inside an acoustic chamber. Calibrated auditory stimuli were amplified and presented via a speaker (Ultrasonic Power Amplifier and Ultrasonic Dynamic Speaker Vifa; Avisoft Bioacoustics, Berlin, Germany), which was centrally located in the ceiling of the chamber. Background signals consisted of narrowband noise of 10,16 , and $20 \mathrm{kHz}$ at $75 \mathrm{~dB}$. The startle stimulus was a 20-ms-long, 115-dB-equivalent sound pressure-level broad band noise (BBN) burst. A silent gap of $50 \mathrm{~ms}$ was embedded in the background noise, $100 \mathrm{~ms}$ before the startle stimulus in all gap trials. One session consisted of 10 gap trials and 10 startle-only trials for every background sound. Before each session, subjects were acclimatized for $5 \mathrm{~min}$ and habituated by presenting 10 startle-only trials. To 
calculate the gap:no gap ratios, amplitude of each gap-startle was divided by the corresponding mean of no-gap startles. Two complete sessions per condition were performed for each subject on separate consecutive days. One additional complete session was performed at the start of the experiment to habituate the animals to the testing procedure.

\section{Single Unit Recordings}

Electrophysiological measurements were conducted 4-5 wk after noise exposure. The order of the experiments in the two study groups was randomized. Rats were anesthetized with urethane $(1.5 \mathrm{~g} / \mathrm{kg}$ ip) and fixed in a stereotactic frame (model 51950, Stoelting Co., Wood Dale, IL) with hollow ear bars (World Precision Instruments, Sarasota) to allow presentation of auditory stimuli. Body temperature was controlled and maintained at $37^{\circ}$ with a heating pad (ATC1000, World Precision Instruments, Sarasota, FL). To allow access to the MGB, a small craniotomy and durotomy was performed contralateral to the side of noise exposure at identified coordinates (22). A glass microelectrode, pulled from Kwik-Fil (World Precision Instruments, Sarasota, FL) filamented borosilicate glass capillaries with a tip diameter of $1 \mu \mathrm{m}$, containing $0.9 \% \mathrm{NaCl}$, was then carefully lowered into the MGB (AP $-5.4-5.8 \mathrm{~mm}$, ML 3.4-3.5 mm relative to bregma, DV 3.8$6.8 \mathrm{~mm}$ from dura) using a hydrolic drum Microdrive (FHC, Bowdoin). The electrode was connected to an AlphaMap data acquisition system (AlphaOmega, Nazareth, Israel), allowing recording of extracellular neuronal activity (sampling rate, $25 \mathrm{kHz}$; high-pass filter, $350 \mathrm{~Hz}$; low-pass filter, $5 \mathrm{kHz}$ ). A sound paradigm was designed using a custommade MATLAB script (V2015A; MathWorks, Inc., Natick, $\mathrm{MA})$. Sounds were processed with an external soundcard (EMU 0204, Creative Technology, Ltd., Singapore) with a sampling rate of $192 \mathrm{kHz}$ and amplified using an Ultrasonic power amplifier and Ultrasonic Dynamic Speaker Vifa (Avisoft Bioacoustics, Berlin, Germany). While lowering the electrode, search stimuli ( $200 \mathrm{~ms}$ BBN, 2 presentations/s) were played. Lowering was stopped each time a well isolated spike was observed. A standardized paradigm of auditory stimuli was presented to determine characteristic frequency and thresholds. This paradigm consisted of 50-ms-long stimuli (2 presentations/s) with frequencies from $1 \mathrm{kHz}$ to $32 \mathrm{kHz}$ in steps of 1 octave (pseudorandomly presented) and intensities from $100 \mathrm{~dB}$ to $10 \mathrm{~dB}$ in steps of $10 \mathrm{~dB}$. Next, 100 repeats of $200 \mathrm{~ms}$ and $95 \mathrm{~dB}$ SPL BBN stimuli with two presentations per second were presented to determine response type. Lastly, spontaneous firing was recorded for $5 \mathrm{~min}$. Per animal, multiple trajectories were performed. Unstable neurons and neurons lost during recording were excluded from further analyses.

\section{Local Field Potentials and Electroencephalography}

After removal of the glass microelectrode, a bipolar electrode was introduced in the MGB (AP $-5.7 \mathrm{~mm}$, ML $3.9 \mathrm{~mm}$, and DV $-6 \mathrm{~mm}$ relative to bregma). This coaxial gold-coated electrode had a platinum-iridium inner wire, shaft diameter of $250 \mu \mathrm{m}$ and tip diameter of $\sim 50 \mu \mathrm{m}$ (23). In addition, a cortical electrode consisted of a miniature screw at the vertex, with subcutaneous reference wire, placed on the left mastoid bone. Electrodes were connected to a data-acquisition system
(PowerLab 8/35, New South Wales, Australia). Signals were sampled at $20.000 \mathrm{~Hz}$ and recorded using LabChart Pro 7 software (ADInstruments, Castle Hill, Australia), high-pass filter was set at $0.1 \mathrm{~Hz}$, and low-pass filter at $1 \mathrm{kHz}$. Two periods of $5 \mathrm{~min}$ of data were recorded before and directly after DBS.

\section{Deep Brain Stimulation}

High-frequency stimulation was applied between LFP measurements. Therefore, the bipolar electrode was connected to a constant-current isolator (DLS 100; WPI, Berlin, Germany), which was connected to a stimulator (DS8000; World Precision Instruments, Sarasota, FL). Subjects were stimulated for 5 min continuously. Stimulation parameters were based on previous experiments (19): frequency of $100 \mathrm{~Hz}, 60 \mu \mathrm{s}$ pulse width, and $100 \mu \mathrm{A}$ amplitude. Monophasic pulses were applied.

\section{Tissue Collection and Immunohistochemistry}

At the end of electrophysiological recordings, rats were decapitated, and brains were quickly removed and frozen in $-40^{\circ} \mathrm{C}$ 2-methyl-butane (isopentane). Brains were serially cut with a cryostat (Leica CM3050S, Nussloch, Germany) in $50-\mu \mathrm{m}$-thick sections. Standard hematoxylin-eosin staining was performed to evaluate the exact location of tips of stimulation and LFP recording electrodes.

\section{Data Analyses}

MATLAB software (V2015A; MathWorks, Inc., Natick) with custom-written codes was used. Only recordings that showed stable firing were included for further analysis. Spike thresholds were manually selected per data set by visual and auditory inspection. Spike waveforms were sorted using principal component analysis and $\mathrm{K}$ means clustering, to determine one or multiple single units per recording. Clusters representing single units were selected and confirmed by inspection of their autocorrelation.

All sorted single units were automatically divided into four main response types, based on the PSTH in response to BBN stimuli. Criteria for this categorization were based on previous literature $(24,25)$. A neuron was considered a "fast" response type if two criteria were met: 1) the response rate within $50 \mathrm{~ms}$ after the stimulus onset was more than 2 standard deviations higher than its spontaneous firing rate, and 2) the response within $50 \mathrm{~ms}$ after stimulus onset rate was more than $30 \%$ higher than the firing rate $50-100 \mathrm{~ms}$ after stimulus onset. If only the first criterion was met, the neuron was considered a "sustained" response type. In case the response rate within $100 \mathrm{~ms}$ after stimulus onset was at least 1 standard deviation lower than the spontaneous firing rate, it was considered a "suppressed" neuron. All other units that did not show a response on BBN stimuli were assigned to the "no response" group.

To quantify spike irregularity, coefficient of variation (CV) was calculated per unit by dividing the standard deviation of inter spike intervals by the mean. Best frequency of each unit was defined as the frequency that corresponds to the highest firing rate within $50 \mathrm{~ms}$ after stimulus onset for the highest two intensities (90-100 dB). For suppressed response types, we took the lowest firing rate (i.e., the strongest suppression). In case two or more stimulus conditions had the 
same response, we took the best frequency as the average of these frequencies. Response rate heatmaps per stimulus intensity were computed for the best frequency of each unit. Response rate-level functions were calculated for each neuron's best frequency, $16 \mathrm{kHz}$ (the frequency used for noise trauma), and $1 \mathrm{kHz}$ stimulus (far from the affected frequency). To determine $50 \%$ thresholds $\left(\mathrm{L}_{50}\right)$, a Naka-Rushton function $(26,27)$ was fitted to the mean response at each stimulus level. The form of the fitted function was as follows:

$$
Y=\left(\mathrm{R}_{\max } \times \frac{X^{n}}{\mathrm{~L}_{50^{n}}+X^{n}}\right)+\mathrm{R}_{\min },
$$

where $Y$ is the predicted response to a stimulus of level $X, \mathrm{R}_{\max }$ is the maximum response, and $\mathrm{L}_{50}$ is the stimulus intensity which produces a response equal to $50 \%$ of the difference between $R_{\max }$ and the spontaneous activity $R_{\min }$, which we took to be the $50 \%$ threshold. The exponent $n$ is proportional to the slope of the curve at the intensity $\mathrm{L}_{50}$, for ease of interpretation this was transformed to units of spikes/s/DB. The four free parameters, $\mathrm{R}_{\max }, \mathrm{L}_{50}$, $\mathrm{R}_{\min }$, and $n$ were optimized by minimizing the summed squared error using MATLAB's Fminsearch function. In addition, we calculated a statistical threshold $\mathrm{L}_{\text {th }}$ as level, where the fitted-firing rate was one SD above the minimum rate $R_{\min }$ (28). Response dynamic range was calculated as the difference between $\mathrm{L}_{\text {th }}$ and the saturating level $\mathrm{L}_{\text {sat }}$, where the fitted rate is one $\mathrm{SD}$ below $\mathrm{R}_{\max }(29)$.

Fit quality was assessed be the percentage of variance accounted for by the fitted model (30) calculated as:

$$
\% \text { Variance }=100 \times\left(1-\begin{array}{r}
D(m, r) \\
D(R, r)
\end{array}\right),
$$

where $D(m, r)$ corresponds to the mean squared difference between the model predicted response ( $m$, see $E q .1)$ and the observed mean firing rate $(r)$ at each level and $D(R, r)$ corresponds to the mean squared difference between the grand mean firing rate $(R$, calculated across stimulus levels) and mean firing rate at each stimulus, separately.

LFP data were analyzed using the fieldtrip MATLAB toolbox (31) and custom-written scripts. To remove line noise, a notch filter was applied at 50, 100, and $150 \mathrm{~Hz}$. Furthermore, for power analysis the data were decomposed using singlespectrum decomposition $(32,33)$ and the component corresponding to $50 \mathrm{~Hz}$ was removed before recombining the data. Data were then down sampled to $250 \mathrm{~Hz}$ and were high-pass filtered above $0.7 \mathrm{~Hz}$. Sections of data containing artifacts were visually identified and rejected. Data were cut into nonoverlapping epochs of $1 \mathrm{~s}$ and power and cross-spectrum were calculated using a multitaper method with discrete prolate spheroid sequences (DPSS) for frequencies of 1 to $100 \mathrm{~Hz}$ (smoothing $\pm 4 \mathrm{~Hz}$ ). Connectivity was estimated by calculating debiased weighted phase lag index (dwPLI) from these cross spectra (34). To test for recovery after DBS, data were separated into five bins representing 1-min time intervals of the 5 min following DBS.

\section{Statistical Analyses}

For GPIAS analysis, Wilcoxon signed rank test was used and Bonferroni-Holm corrected $P$ values are presented. Analysis of single units was performed using the following tests. A $\chi^{2}$ test of goodness of fit was performed to compare distribution of the four response types between groups. Permutation tests with 1,000 randomizations were used to test for differences in spontaneous firing rate (SFR), CV, $\mathrm{L}_{50}$, $R_{\min }, R_{\max }$, slope, $L_{t h}$, and dynamic range between groups. To determine differences in rate-level functions, a mixed ANOVA was performed, including one between-subjects factor "group" (2 levels, noise-exposed or unexposed) and one within-subject factor "stimulus intensity" (10 levels, 10 $-100 \mathrm{~dB}$ ). Cluster analysis of spike waveforms obtained from the Gaussian mixture models was performed to test for differences between response types. One-way ANOVA was performed to find a relation between response types and recording coordinates in three dimensions. For LFP analysis, mixed ANOVA with "group" as between-subjects factor and "time" (6 levels, baseline and 1-5 min post-DBS) as withinsubjects factor were performed to test for differences in phase synchronization. A significance level $\alpha$ for all statistical tests was 0.05. Analysis of behavioral data was performed with SPSS (v. 22.0 for Mac, SPSS, Chicago, IL). MATLAB Software (R2015a, 64-bit) was used to analyze electrophysiological data.

\section{RESULTS}

\section{Gap Prepulse Inhibition of Acoustic Startles}

Results of GPIAS are presented in Fig. 1. Gap:no gap ratios were increased in the noise-exposed group for the 16 and $20 \mathrm{kHz}$ background sound $(\mathrm{Z}=2.55, P=0.01$ and $\mathrm{Z}=2.66, P=$ 0.01 , respectively), but not for $10 \mathrm{kHz}(\mathrm{Z}=1.24, P=0.21)$. The control group did not show altered gap:no gap ratios $(\mathrm{Z}=$ $-0.14, P=0.89$ for $10 \mathrm{kHz}, \mathrm{Z}=-1.21, P=0.23$ for $16 \mathrm{kHz}$, and $\mathrm{Z}=-1.48, P=0.14$ for $20 \mathrm{kHz}$ ). These GPIAS responses are similar to earlier published studies $(19,21,35)$ and indicate existence of tinnitus in the noise-exposed group. Our setup with freely moving subjects did not allow to validly discriminate individual tinnitus positive and negative animals, therefore we did not exclude subjects and labeled our group as "noise-exposed."

\section{Single Unit Recordings}

In total, 214 single units were identified [ $n=145$ (average of 16.1 per subject) in the noise-exposed group, $n=69$ (average of 13.8 per subject) in unexposed control group]. Based on PSTH during BBN stimuli, four response types were distinguished (Fig. 2A): $I$ ) fast response ( $n=28$ for the noise-exposed group and $n=29$ for unexposed controls), II) sustained response $(n=28$ and $n=11), I I I)$ suppressed response $(n=27$ and $n=10)$, and $I V)$ no response $(n=62$ and $n=19$ ). Most of the units of group IV were found in the middle of a trajectory with adjacent responding neurons, suggesting these responses were likely within the MGB. The proportions of response types differed between the noise-exposed group and the unexposed control group, $\chi^{2}$ $(3, n=214)=12.68, P=0.005$ (Fig. $2 B)$. Notably there was a higher proportion of nonresponsive units and a lower proportion of fast-responsive neurons in the noise-exposed group compared with the control group, whereas the proportions of sustained and suppressed neurons were similar in both groups. 
Unexposed

Baseline

After sham exposure

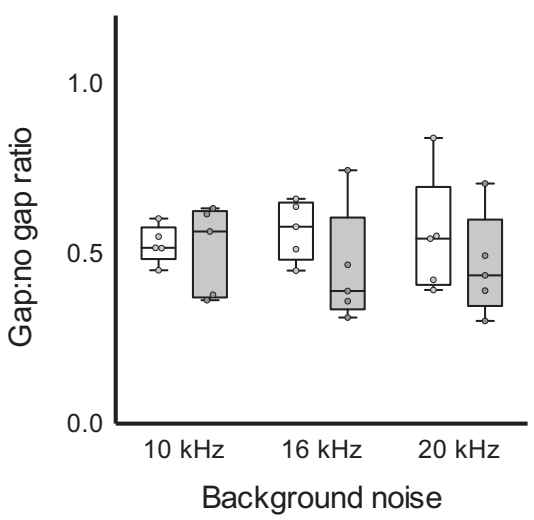

\section{Noise-exposed}

$\square$ Baseline

After $16 \mathrm{kHz}$ exposure

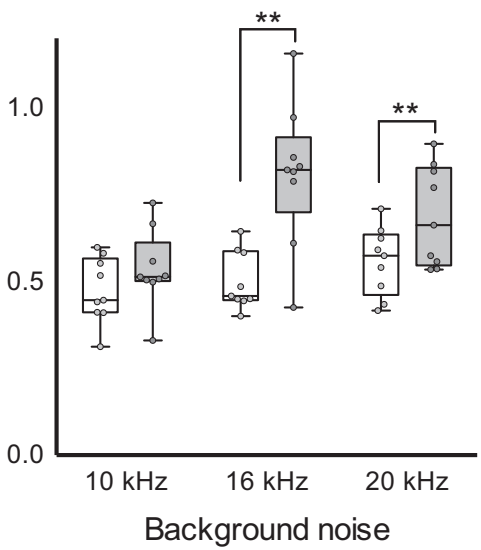

Figure 1. Gap:no gap ratios of both study groups. Detection of the gap was significantly reduced after $16 \mathrm{kHz}$ noise exposure for the 16 and $20 \mathrm{kHz}$ background sound, $* * P<$ 0.01 .
Characteristics of single unit recordings during 5-min spontaneous recording are presented in Table 1. Overall, there was increased spontaneous firing in the noiseexposed subjects (mean SFR of $9.65 \pm 0.7$ and $7.40 \pm 0.9, P=$ 0.03). Subgroup analysis revealed that the suppressedresponse-type neurons showed a significantly higher spontaneous firing rate in the noise-exposed group (mean of $15.06 \pm 1.4$ spikes/s, compared with $9.68 \pm 2.1$ spikes $/ \mathrm{s}, P$
$=0.006$. An increase was also observed in the sustained response type, which almost reached significance (mean $9.77 \pm 2.2$ and $4.20 \pm 1.9$ spikes $/ \mathrm{s}, P=0.052$ ). In the fastresponding and no-responding neurons, there were no differences. There was no significant difference in CV between the two groups.

The sensitivity of individual neurons was characterized by fitting a Naka-Rushton function $(26,27)$ to response rates at
A
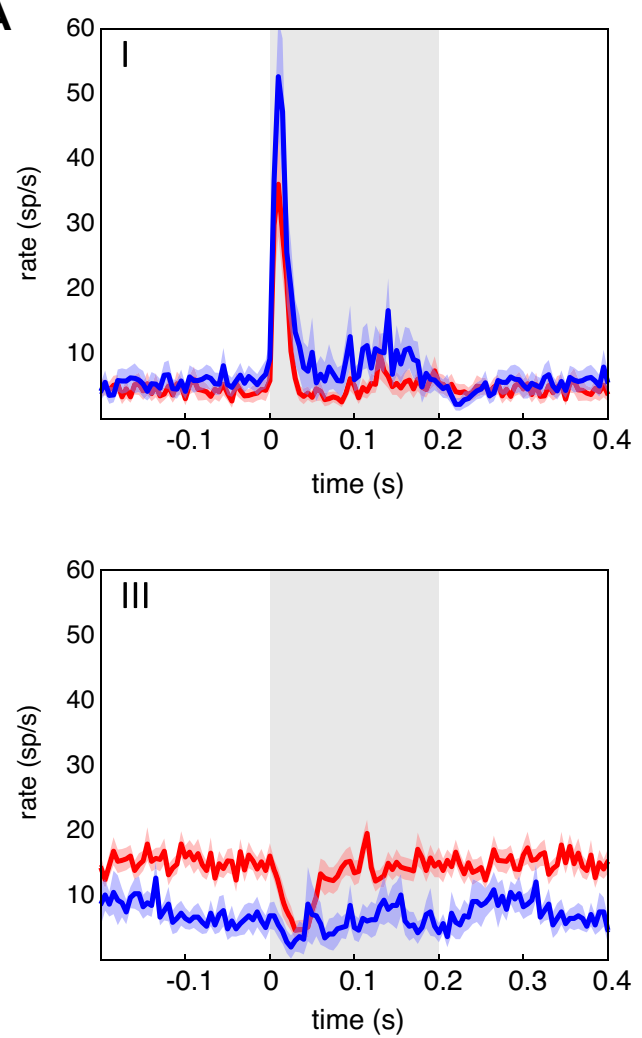
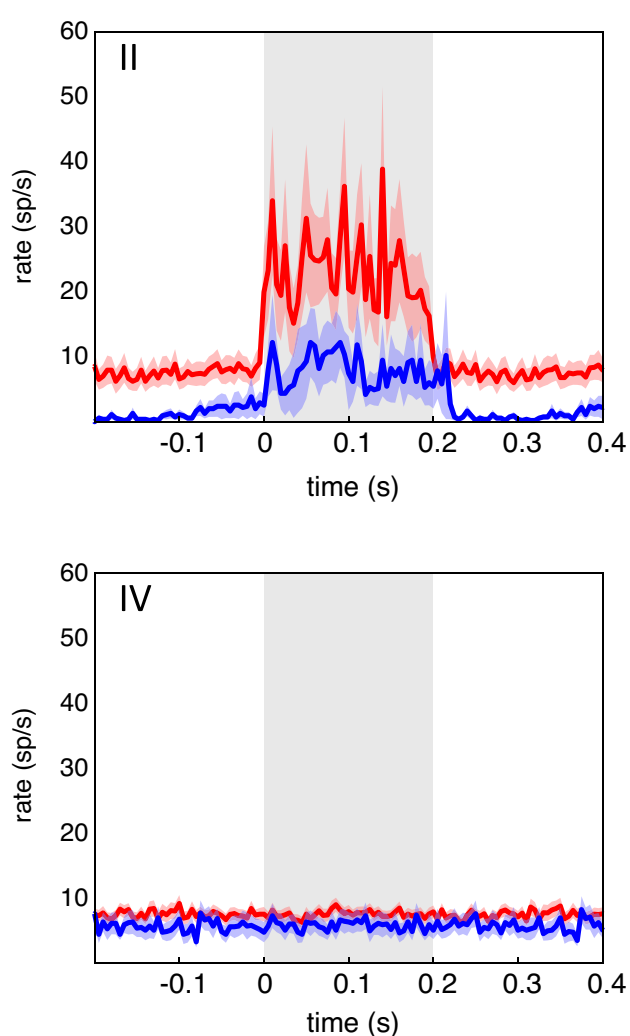

B
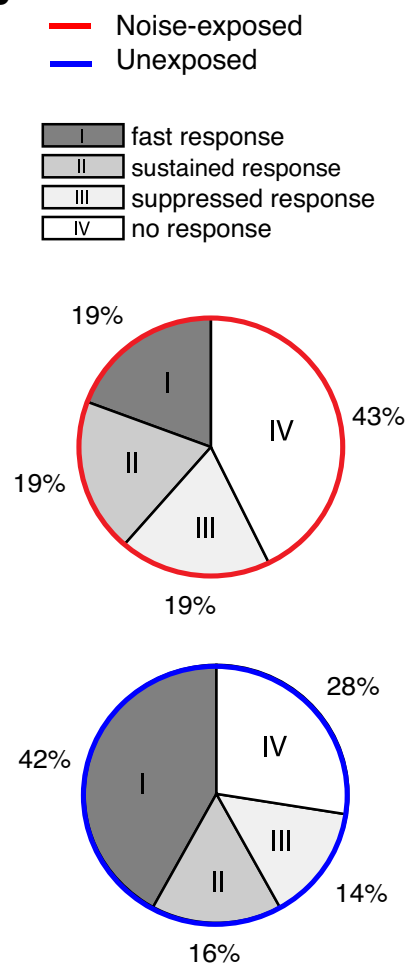

Figure 2. A: PSTH of all single unit recordings during broad band noise (BBN) stimuli, divided in four response types: I) fast response, II sustained response, III) suppressed response, and $I M$ no response. Solid lines show means response, shading shows \pm SE. B: proportions of response types differed per study group. $n=145$ single-unit recordings in noise-exposed subjects, $n=69$ in unexposed controls. 
Table 1. Characteristics of all single unit responses

\begin{tabular}{|c|c|c|c|c|c|c|c|c|}
\hline & \multicolumn{2}{|c|}{ Number of Units } & \multicolumn{3}{|c|}{ Spontaneous Firing (Spikes/s) } & \multicolumn{3}{|c|}{ Coefficient of Variation } \\
\hline All responses & 145 & 69 & $09.65 \pm 0.7$ & $7.40 \pm 0.9$ & $0.03 *$ & $1.09 \pm 0.04$ & $1.18 \pm 0.07$ & 0.16 \\
\hline Sustained & $28(19 \%)$ & $11(16 \%)$ & $09.77 \pm 2.2$ & $4.20 \pm 1.9$ & 0.05 & $1.26 \pm 0.11$ & $1.73 \pm 0.29$ & 0.08 \\
\hline Suppressed & 27 (19\%) & 10 (14\%) & $15.06 \pm 1.4$ & $9.68 \pm 2.1$ & $0.006 * *$ & $0.86 \pm 0.07$ & $0.92 \pm 0.12$ & 0.39 \\
\hline No & 62 (42\%) & 19 (28\%) & $8.61 \pm 0.9$ & $6.08 \pm 1.2$ & 0.10 & $1.07 \pm 0.05$ & $0.95 \pm 0.08$ & 0.81 \\
\hline
\end{tabular}

Data are presented as means \pm SE. Permutation testing was performed on all single unit responses, with additional post hoc tests per response type. $* P<0.05, * * P<0.01$.

10 sound intensities. Here, we considered the units where the fit quality exceeded an explained variance of $70 \%$. Physiological parameters of the rate-level functions are presented in Table 2. Thresholds ( $\mathrm{L}_{50}$ and $\mathrm{L}_{\mathrm{th}}$ ) of fast-responding units were significantly higher, indicating lower sensitivity, in the noise-exposed group. The other response types did not show altered $\mathrm{L}_{50}$ or $\mathrm{L}_{\mathrm{th}}$ thresholds. The other parameters, $R_{\min }, R_{\max }$, and slope and dynamic range, did not statistically differ between groups.

Surface plots and rate-level functions during frequency tuning measurements are shown per response type in Fig. 3. Visual inspection of the surface plots (Fig. $3 A$ ) reveals two main differences between the noise-exposed and unexposed control group. First, spontaneous firing of the sustained and suppressed neurons was higher in the noise-exposed group, in line with results from Table 1. Second, for fastresponding neurons, stimulus-evoked responses were higher particularly for lower intensities in the unexposed group. This is in line with results from Table 2, where the thresholds were higher in the noise-exposed group for this neuron type. For units with a sustained response the pattern was more mixed.

To summarize the surface plots, we calculated rate-level functions from firing rate during $0.05 \mathrm{~s}$ after stimulus onset. Analysis of the rate-level functions of fast-responding cells during presentation of the best frequency (Fig. 3B, first column) revealed a significant interaction between groups (noise-exposed or unexposed control) and stimulus intensity $[F(1,9)=3.23, P=<0.001]$. In unexposed controls, higher stimulus intensities led to higher firing rate compared with the noise-exposed group [main group effect $F(1,55)=6.74, P=$ 0.01 ). Interestingly, for the sustained cells, there were no significant differences between groups. The suppressed response type showed a difference in firing rate between groups regardless of stimulus intensity [main group effect $F(1,35)=4.83, P=$ 0.03]. Analysis of responses to the $16 \mathrm{kHz}$ noise-trauma frequency (Fig. 3B, second column) revealed different results. In both fast- and sustained-responding neurons, no effect of noise trauma was found. Notably, there was a significant group difference for the suppressed neurons $[F(1,35)=4.54, P=$ 0.04], which was independent of intensity of the stimuli. For the $1 \mathrm{kHz}$ control frequencies, there were no significant group differences found for any response type.

All supplemental material is available at https://doi.org/ 10.6084/m9.figshare.12662672.

\section{Local Field Potentials and Electroencephalography}

\section{Electrode localization.}

After recording single unit activity, the microelectrode was replaced with a DBS probe capable of recording LFP and providing electrical stimulation. The DBS electrode positions used for LFP recording and high-frequency stimulation are visualized in Fig. 4.

\section{Phase synchronization and power.}

Phase synchronization between MGB and cortex and power were analyzed at baseline (5 $\mathrm{min}$ ) and in 1-min time intervals after high-frequency stimulation of the MGB (Fig. 5A). Statistical analysis of thalamocortical phase synchronization revealed significant main effects of DBS (time) at the $\beta$ frequency band $[20-35 \mathrm{~Hz}, F(1,5)=4.90, P<0.001)$ and $\gamma$ frequency $[50-70 \mathrm{~Hz}, F(1,5)=3.02, P=0.02]$. All effects were independent of group, as there were no significant interactions. For $\delta(1-4 \mathrm{~Hz}), \theta(4-8 \mathrm{~Hz})$, and $\alpha(8-12 \mathrm{~Hz})$ bands, no significant interactions or main effects were observed. Analysis of power spectra showed a significant suppressing effect of DBS on all tested frequency bands, without a group effect (Fig. 5B) in the MGB electrode. For all frequency bands, DBS led to a brief increase in power, after which it decreased to levels lower than baseline $[\delta F(1,5)=8.89, P<$ $0.001 ; \theta F(1,5)=7.42, P<0.001 ; \alpha F(1,5)=6.24, P<0.001 ; \beta F$ $(1,5)=3.02, P=0.02$, and $\gamma F(1,5)=3.67, P=0.01]$. At the cortical level, power at these frequency bands did not significantly change after application of DBS.

\section{DISCUSSION}

In this study, we examined neurophysiological characteristics of the MGB and the effects of DBS of the MGB in the noise-exposed animal model. Our main findings are first, a reduction in the population of fast-responding neurons and a corresponding increase in nonresponsive neurons after noise exposure. Second, acoustic overexposure leads to an increased spontaneous firing of sustained- and suppressedresponse-type neurons without influencing the responsiveness to stimuli. Third, fast-responding neurons in noiseexposed subjects showed an increased threshold without a change in the spontaneous firing rate. In addition, we found that MGB DBS suppressed thalamocortical synchronicity in both noise-exposed and control animals.

\section{Neurophysiological Hallmarks of the MGB in the Noise- Exposed Tinnitus Animal Model}

Spontaneous firing rates were increased in the noiseexposed group. This finding was in line with reports by others in awake (16), but not in anesthetized (17) tinnitus animals. Although previous reports did not differentiate between neuron types, our results show that this increase in spontaneous firing was only present in a subtype of MGB 


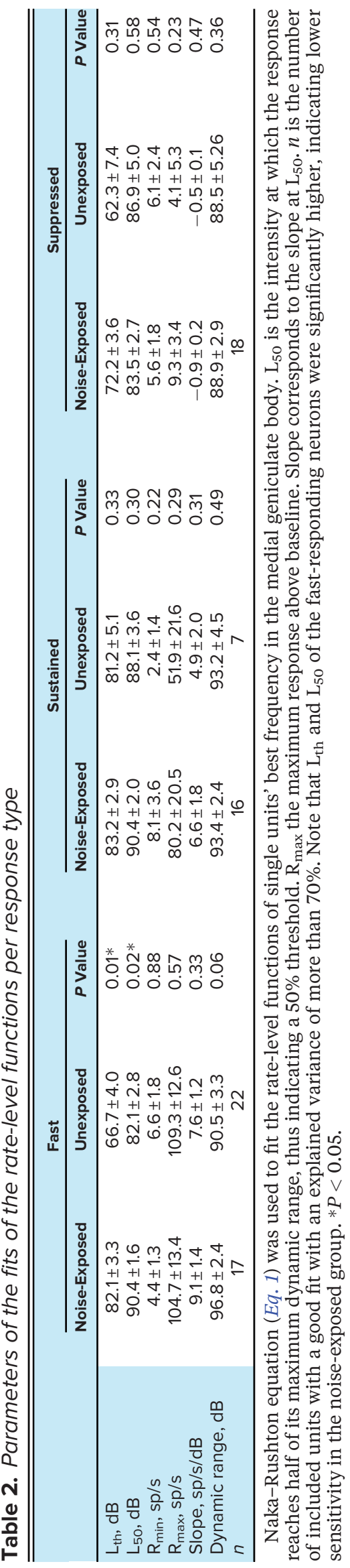

neurons: only the suppressed- and sustained-responsetype neurons but not fast-responding and nonresponding neurons. Interestingly, in the noise-exposed animals, the number of fast-responding neurons was reduced, whereas there was an increase in the proportion of nonresponding neurons. The proportion of sustained- and suppressed-responding neurons remained the same. The question arises of whether specific response types belong to different excitatory or inhibitory neuronal cell types. We could not find differences in waveform, nor location of recording. In the rat $\mathrm{MGB}$, there are morphologically different cell types (36). GABAergic neurons are in low abundance, likely less than $1 \%$ in the MGB of the rat (37) and most recorded neurons are probably glutamatergic. The increased spontaneous activity of sustained- and suppressed-response types might therefore reflect an increase in excitatory neuronal activity. Hyperactivity of sustained and suppressed neurons might be the readout at a single neuron level of thalamocortical dysrhythmia as described by other electrophysiological studies at the population and network level $(2,38)$.

The undisputed connection between tinnitus, hearing loss, and hyperacusis is a major challenge in the field of tinnitus research (1). These symptoms often co-exist and share similar etiological and pathophysiological factors. It has recently been postulated that there is a certain degree of hearing loss in almost all human and animal tinnitus cases, which is sometimes undetectable by audiograms or auditory brainstem responses $(39,40)$. A limitation of our study is that we only compared noiseexposed animals to sham-exposed controls. Our results confirmed the existence of tinnitus-like behavior on a group level, but our setup did not allow to validly differentiate tinnitus positive or negative animals on an individual level. Therefore, it remains uncertain what the confounding effects of these two symptoms are in our findings.

Based on our findings, it might be postulated that different characteristics of response types reflect different symptoms. We propose that tinnitus perception might be due to increased spontaneous activity of sustained and suppressed neurons, possibly contributing to increased oscillations and thalamocortical dysrhythmia. Hearing loss could be reflected by the increase in the proportion of nonresponding neurons, together with the loss of fast-response-type neurons and their increased thresholds. Hyperacusis might be related to an increased response to external stimuli, which has been shown before (16). In our study, we found an increased response in sustained neurons, but this difference did not reach significance between groups. These hypotheses definitely need further validation. It is tempting to speculate that loss of fast-responding neurons is causally linked to the increase in spontaneous firing rate of sustained and suppressed neurons. We, however, did not find a correlation between the two factors, which might be due to the small sample size. Additional electrophysiological studies in both animal models and humans would be valuable and might relate specific properties of the MGB neural subtypes to hearing loss, hyperacusis, and tinnitus. 
A

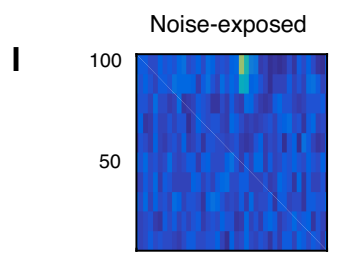

II

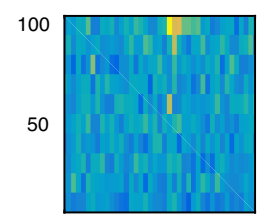

III

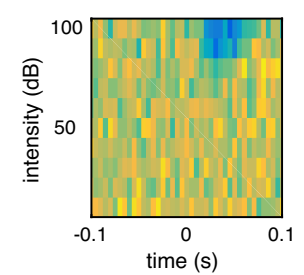

Unexposed
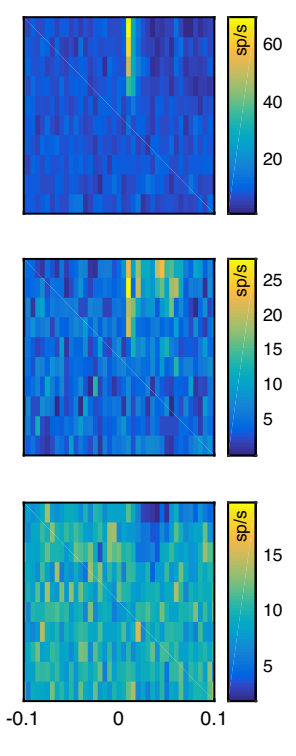

B
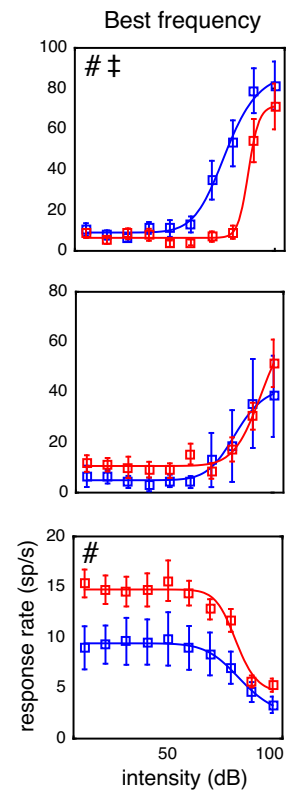

- Noise-exposed

Unexposed
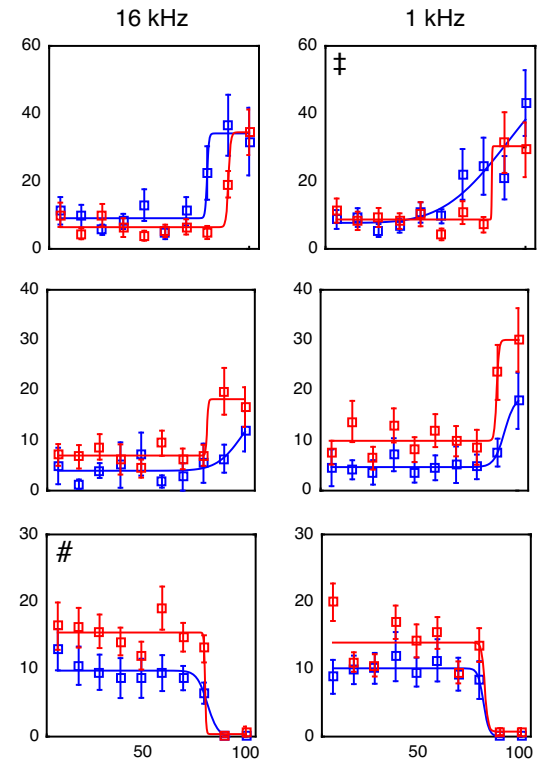

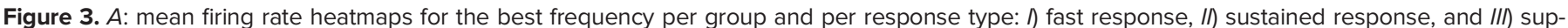

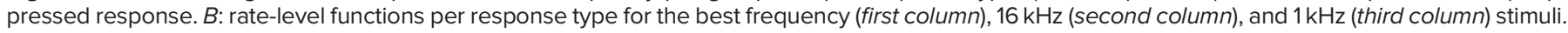

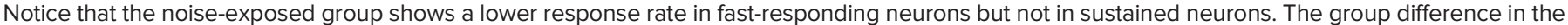

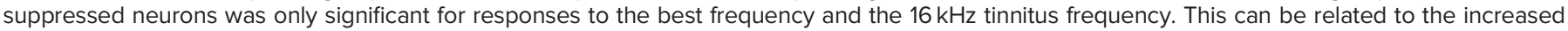

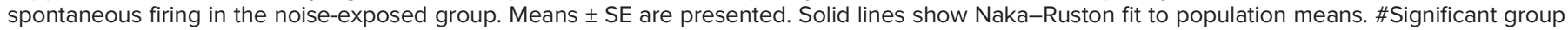
effect (noise exposed vs. unexposed control). †Significant interaction (group $\times$ intensity).

Thalamocortical dysrhythmia has been proposed as an underlying mechanism of tinnitus $(2,11)$. In our study, no differences in power and corticothalamic synchronization between noise-exposed tinnitus animals and controls were found. Thalamocortical dysrhythmia and synchronization by loss of inhibition has been shown in the salicylateinduced tinnitus model. An increased cortical $\gamma$ activity was proven to be associated with enhanced $\theta-\gamma$ coupling, as well as a decreased $\alpha$ power and coherence between the auditory cortex and the MGB (38). Our negative finding might be due to a small sample size, as our study was not powered to find group effects for LFP recordings. To avoid type 2 error, firm conclusions cannot be drawn on the effect of noise trauma on thalamocortical synchronization based on our study.

\section{Deep Brain Stimulation of the MGB}

Previously, it has been shown that MGB DBS suppresses tinnitus-like behavior in rats (19). The current study shows a temporary disrupting effect of MGB DBS on corticothalamic synchronization in the $\beta$ and $\gamma$ band. Furthermore, we found a suppressing effect on the total power of the LFP in all frequency bands after MGB DBS.

The exact working mechanism of DBS remains elusive. An accepted principle is that high frequency DBS causes an inactivation or a temporary lesion of the target area (41). In

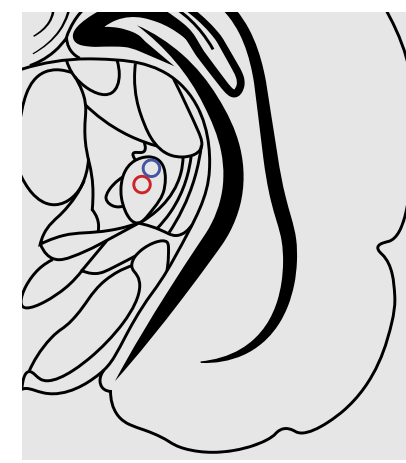

Bregma $-4.8 \mathrm{~mm}$

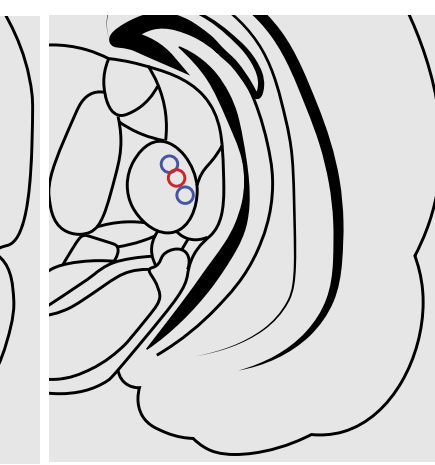

Bregma $-5.2 \mathrm{~mm}$

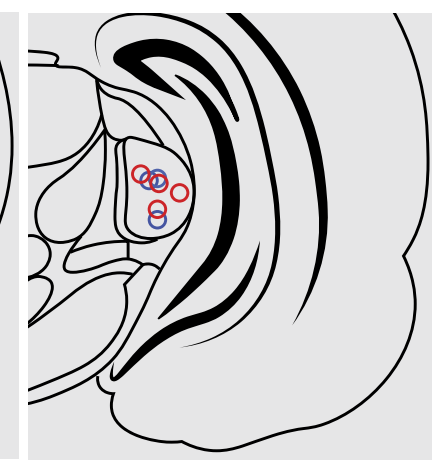

Bregma $-5.6 \mathrm{~mm}$

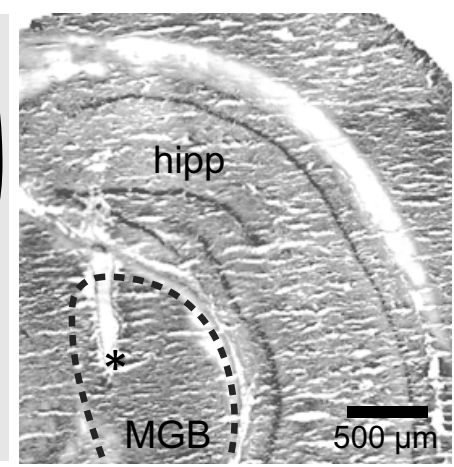

MGB

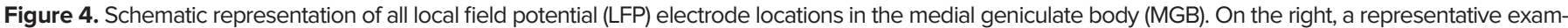

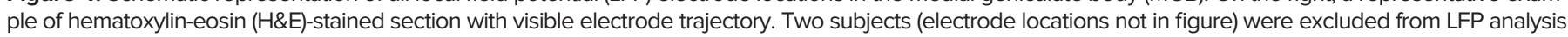
due to incorrect positioning and hardware failure. Noise exposed in red, unexposed control in blue. *Location of the electrode tip. Hipp, hippocampus. 
A

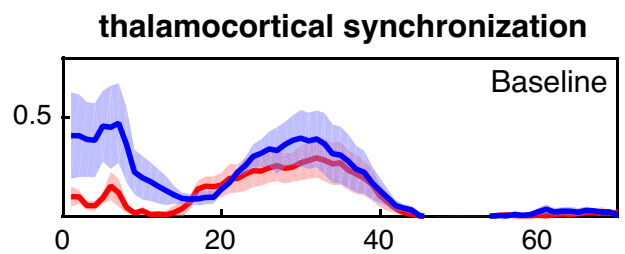

B

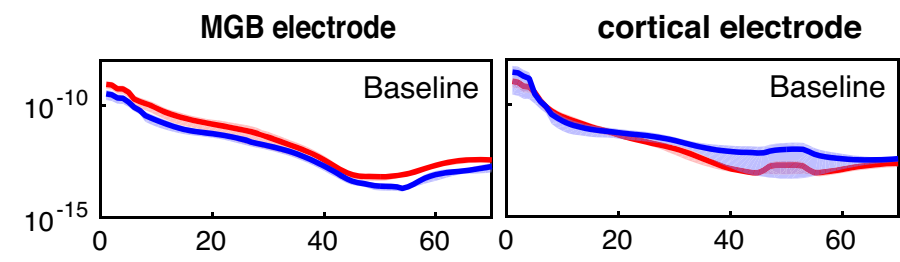

\section{5 minutes DBS}
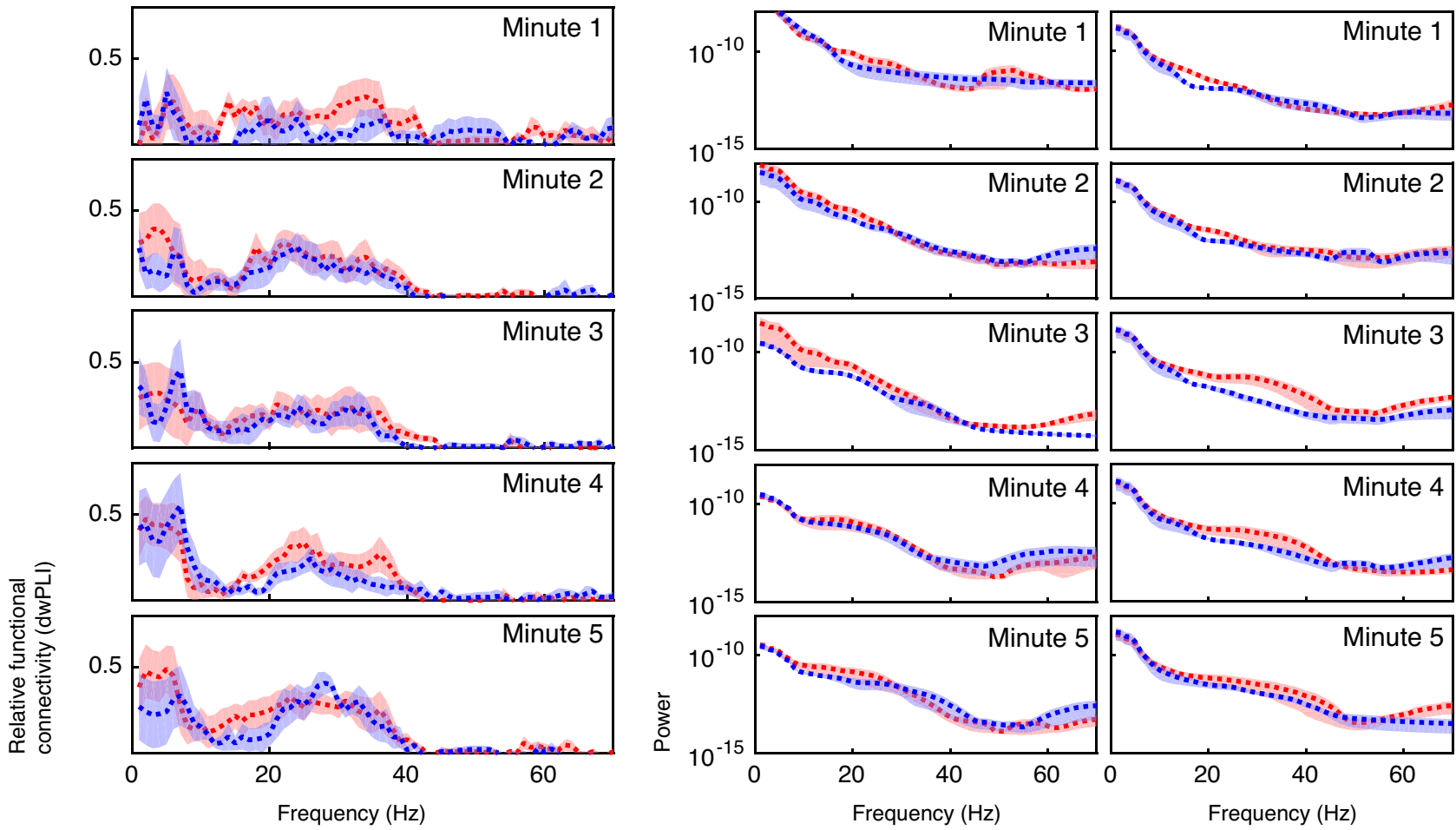

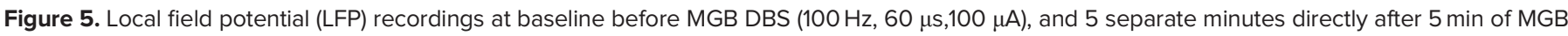

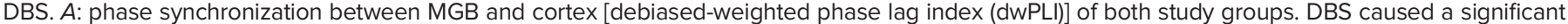

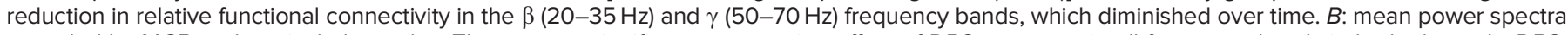

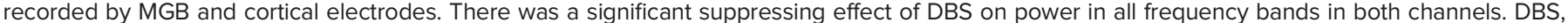
deep brain stimulation; MGB, medial geniculate body.

the noise-exposed animal model as used in the current study, spontaneous hyperactivity within the MGB could well be related to tinnitus. MGB DBS might suppress this aberrant activity. Other studies report a downregulation of inhibitory neurotransmission in the inferior colliculus in tinnitus (4244). High-frequency DBS at the MGB could cause presynaptic GABA release of these projections and herewith suppress MGB activity. Furthermore, stimulation might suppress cell body activity by inducing a depolarization block. Besides a local inhibitory effect, DBS also causes distant excitatory actions. Direct stimulation of axons nearby the electrode can induce tonic patterns of action potential in structures that are in a distance from the stimulated target. Firing characteristics of postsynaptic cells might be influenced by multiple underlying effects, such as effects on neurotransmitter release, changes in metabolic activity, and plastic changes such as long-term potentiation and depression (41). Considering these complex distant effects, activation of reticular nucleus neurons by MGB DBS might influence thalamic gating function in tinnitus. Overall, aforementioned mechanisms might lead to increased thalamocortical $\gamma$ oscillations, which have been associated with tinnitus (45). Our findings support the theory that MGB DBS blocks abnormal information flow, such as thalamocortical dysrhythmia, as we found a desynchronizing effect at the higher frequencies after MGB DBS.

In line with our previous behavioral study (19), the effect of MGB DBS on synchronization was residual, as corticothalamic synchronization in the $\beta$ and $\gamma$ bands was initially suppressed, but returned approximately to baseline levels within 5 min. MGB DBS can thus have neurophysiological 
effects that extend the stimulation period and may induce neural plasticity. This finding is important for future clinical application of MGB DBS in humans. Stimulation could be performed in ON and OFF cycles, resulting in a longer battery life and reduction of stimulation-induced side effects and habituation effects.

\section{CONCLUSION}

The MGB has a prominent role in auditory pathophysiology such as tinnitus. Based on single unit recordings, we propose a distinct role for separate functional classes of neurons affected by noise trauma, whereby fast-responding neurons become less responsive or nonresponsive without change to their spontaneous rate, whereas sustained- and suppressedtype neurons exhibit enhanced spontaneous activity without change to their stimulus-driven activity. It seems plausible that these findings are strongly correlated to tinnitus. In this light, our LFP findings support suppression of thalamocortical synchronization as an underlying mechanism of tinnitus suppression by high-frequency MGB DBS. Further electrophysiological studies are needed to investigate the role of different neural response types in neuropathophysiology of auditory trauma, as well as the possible effects of MGB DBS on thalamocortical dysrhythmia and thalamic gating function.

\section{GRANTS}

This work was supported by the Dutch Heinsius-Houbolt Foundation.

\section{DISCLOSURES}

The salary of the corresponding author was supported by the Dutch Heinsius-Houbolt Foundation. This is an independent foundation, and this support causes no conflict of interest.

\section{AUTHOR CONTRIBUTIONS}

G.v.Z, Y.T., and M.L.F.J. conceived and designed research; G.v.Z. and F.L.V.W.S. performed experiments; G.v.Z. and M.J.R. analyzed data; G.v.Z., M.J.R., and M.L.F.J. interpreted results of experiments; G.v.Z. prepared figures; G.v.Z., M.J.R., and M.L.F.J. drafted manuscript; F.L.V.W.S. and J.V.S. edited and revised manuscript; G.v.Z., M.J.R., F.L.V.W.S., Y.T., and M.L.F.J. approved final version of manuscript.

\section{REFERENCES}

1. Shore SE, Wu C. Mechanisms of noise-induced tinnitus: insights from cellular studies. Neuron 103: 8-20, 2019. doi:10.1016/j.neuron. 2019.05.008.

2. De Ridder D, Vanneste S, Langguth B, Llinas R. Thalamocortical dysrhythmia: a theoretical update in tinnitus. Front Neurol 6: 124, 2015. doi:10.3389/fneur.2015.00124.

3. Rauschecker JP, Leaver AM, Muhlau M. Tuning out the noise: limbic-auditory interactions in tinnitus. Neuron 66: 819-826, 2010. doi:10.1016/j.neuron.2010.04.032.

4. Eggermont JJ, Roberts LE. The neuroscience of tinnitus. Trends Neurosci 27: 676-682, 2004. doi:10.1016/j.tins.2004.08.010.

5. Kaltenbach JA. Tinnitus: models and mechanisms. Hear Res 276: 52-60, 2011. doi:10.1016/j.heares.2010.12.003.

6. Roberts LE, Eggermont JJ, Caspary DM, Shore SE, Melcher JR, Kaltenbach JA. Ringing ears: the neuroscience of tinnitus. $J$
Neurosci 30: 14972-14979, 2010. doi:10.1523/JNEUROSCI.4028-10. 2010.

7. Caspary DM, Llano DA. Auditory thalamic circuits and GABAA receptor function: putative mechanisms in tinnitus pathology. Hear Res 349: 197-207, 2017. doi:10.1016/j.heares.2016.08.009.

8. Leaver AM, Renier L, Chevillet MA, Morgan S, Kim HJ, Rauschecker JP. Dysregulation of limbic and auditory networks in tinnitus. Neuron 69: 33-43, 2011. doi:10.1016/j.neuron.2010.12.002.

9. Winer JA, Sally SL, Larue DT, Kelly JB. Origins of medial geniculate body projections to physiologically defined zones of rat primary auditory cortex. Hear Res 130: 42-61, 1999. doi:10.1016/S0378-5955 (98)00217-2.

10. Bartlett EL. The organization and physiology of the auditory thalamus and its role in processing acoustic features important for speech perception. Brain Lang 126: 29-48, 2013. doi:10.1016/j.bandl. 2013.03.003.

11. Llinas RR, Ribary U, Jeanmonod D, Kronberg E, Mitra PP. Thalamocortical dysrhythmia: a neurological and neuropsychiatric syndrome characterized by magnetoencephalography. Proc Natl Acad Sci USA 96: 15222-15227, 1999. doi:10.1073/pnas.96.26. 15222.

12. Lanting $\mathbf{C P}$, de Kleine E, van Dijk P. Neural activity underlying tinnitus generation: results from PET and fMRI. Hear Res 255: 1-13, 2009. doi:10.1016/j.heares.2009.06.009.

13. Muhlau M, Rauschecker JP, Oestreicher E, Gaser C, Rottinger M, Wohlschlager AM, Simon F, Etgen T, Conrad B, Sander D. Structural brain changes in tinnitus. Cereb Cortex 16: 1283-1288, 2006. doi:10.1093/cercor/bhj070.

14. Landgrebe $\mathbf{M}$, Langguth $\mathbf{B}$, Rosengarth K, Braun S, Koch A, Kleinjung T, May A, de Ridder D, Hajak G. Structural brain changes in tinnitus: grey matter decrease in auditory and non-auditory brain areas. Neuroimage 46: 213-218, 2009. doi:10.1016/j.neuroimage. 2009.01.069.

15. Smits $\mathbf{M}$, Kovacs S, de Ridder D, Peeters RR, van Hecke P, Sunaert S. Lateralization of functional magnetic resonance imaging (fMRI) activation in the auditory pathway of patients with lateralized tinnitus. Neuroradiology 49: 669-679, 2007. doi:10.1007/s00234-0070231-3.

16. Kalappa BI, Brozoski TJ, Turner JG, Caspary DM. Single unit hyperactivity and bursting in the auditory thalamus of awake rats directly correlates with behavioural evidence of tinnitus. J Physiol 592: 5065-5078, 2014. doi:10.1113/jphysiol.2014.278572.

17. Barry KM, Robertson D, Mulders W. Changes in auditory thalamus neural firing patterns after acoustic trauma in rats. Hear Res 379: 89-97, 2019. doi:10.1016/j.heares.2019.05.001.

18. Basta D, Goetze R, Ernst A. Effects of salicylate application on the spontaneous activity in brain slices of the mouse cochlear nucleus, medial geniculate body and primary auditory cortex. Hear Res 240: 42-51, 2008. doi:10.1016/j.heares.2008.02.005.

19. van Zwieten G, Janssen ML, Smit JV, Janssen AML, Roet M, Jahanshahi A, Stokroos RJ, Temel Y. Inhibition of experimental tinnitus with high frequency stimulation of the rat medial geniculate body. Neuromodulation 22: 416-424, 2018. doi:10.1111/ner.12795.

20. van Zwieten G, Smit JV, Jahanshahi A, Temel Y, Stokroos RJ. Tinnitus: is there a place for brain stimulation? Surg Neurol Int 7: S125-S129, 2016. doi:10.4103/2152-7806.176134.

21. Smit JV, Janssen ML, van Zwieten G, Jahanshahi A, Temel $\mathbf{Y}$, Stokroos RJ. Deep brain stimulation of the inferior colliculus in the rodent suppresses tinnitus. Brain Res 1650: 118-124, 2016. doi:10. 1016/j.brainres.2016.08.046.

22. Paxinos G, Watson C. The Rat Brain in Stereotaxic Coordinates (7th ed.). New York: Academic Press, 2006.

23. Tan S, Vlamings R, Lim L, Sesia T, Janssen ML, Steinbusch HW, Visser-Vandewalle V, Temel Y. Experimental deep brain stimulation in animal models. Neurosurgery 67: 1073-1079, 2010. doi:10.1227/ NEU.0b013e3181ee3580.

24. Bordi F, LeDoux JE. Response properties of single units in areas of rat auditory thalamus that project to the amygdala. II. Cells receiving convergent auditory and somatosensory inputs and cells antidromically activated by amygdala stimulation. Exp Brain Res 98: 275-286, 1994. doi:10.1007/BF00228415.

25. Calford MB, Webster WR. Auditory representation within principal division of cat medial geniculate body: an electrophysiology study. $J$ Neurophysio/ 45: 1013-1028, 1981. doi:10.1152/jn.1981.45.6.1013. 
26. Albrecht DG, Hamilton DB. Striate cortex of monkey and cat: contrast response function. J Neurophysiol 48: 217-237, 1982. doi: 10.1152/jn.1982.48.1.217.

27. Naka KI, Rushton WA. S-potentials from colour units in the retina of fish (Cyprinidae). J Physiol 185: 536-555, 1966. doi:10.1113/ jphysiol.1966.sp008001.

28. Wen B, Wang GI, Dean I, Delgutte B. Dynamic range adaptation to sound level statistics in the auditory nerve. J Neurosci 29: 1379713808, 2009. doi:10.1523/JNEUROSCI.5610-08.2009.

29. Watkins PV, Barbour DL. Level-tuned neurons in primary auditory cortex adapt differently to loud versus soft sounds. Cereb Cortex 21: 178-190, 2011. doi:10.1093/cercor/bhq079.

30. Carandini M, Heeger DJ, Movshon JA. Linearity and normalization in simple cells of the macaque primary visual cortex. J Neurosci 17: 8621-8644, 1997. doi:10.1523/JNEUROSCI.17-21-08621.1997.

31. Oostenveld R, Fries P, Maris E, Schoffelen JM. FieldTrip: open source software for advanced analysis of MEG, EEG, and invasive electrophysiological data. Comput Intell Neurosci 2011: 156869, 2011. doi:10.1155/2011/156869.

32. Bonizzi P, Karel JD, De Weerd P, Lowet E, Roberts M, Westra R, Meste $\mathbf{O}$, Peeters R. Singular spectrum analysis improves analysis of local field potentials from macaque $\mathrm{V} 1$ in active fixation task. Annu Int Conf IEEE Eng Med Bio/ Soc 2012: 2945-2948, 2012. doi:10.1109/ EMBC.2012.6346581.

33. Bonizzi P, Karel JM, Meste $\mathbf{O}$, Peeters R. Singular spectrum decomposition: a new method for time series decomposition. Adv Adapt Data Anal 06: 1450011, 2014. doi:10.1142/S1793536914500113.

34. Vinck M, Oostenveld R, van Wingerden M, Battaglia F, Pennartz CM. An improved index of phase-synchronization for electrophysiological data in the presence of volume-conduction, noise and sample-size bias. Neuroimage 55: 1548-1565, 2011. doi:10.1016/j. neuroimage.2011.01.055.

35. van Zwieten G, Jahanshahi A, van Erp ML, Temel Y, Stokroos RJ, Janssen MLF, Smit JV. Alleviation of tinnitus with high-frequency stimulation of the dorsal cochlear nucleus: a rodent study. Trends Hear 23: 2331216519835080, 2019. doi:10.1177/2331216519835080.
36. Clerici WJ, Coleman JR. Anatomy of the rat medial geniculate body: I. cytoarchitecture, myeloarchitecture, and neocortical connectivity. J Comp Neuro/ 297: 14-31, 1990. doi:10.1002/cne.902970103.

37. Winer JA, Larue DT. Evolution of GABAergic circuitry in the mammalian medial geniculate body. Proc Natl Acad Sci USA 93: 30833087, 1996. doi:10.1073/pnas.93.7.3083

38. Vianney-Rodrigues $\mathbf{P}$, Auerbach BD, Salvi R. Aberrant thalamocortical coherence in an animal model of tinnitus. J Neurophysiol 121: 893-907, 2019. doi:10.1152/jn.00053.2018.

39. Kujawa SG, Liberman MC. Adding insult to injury: cochlear nerve degeneration after temporary noise-induced hearing loss. J Neurosci 29: 14077-14085, 2009. doi:10.1523/JNEUROSCI.284509.2009.

40. Weisz N, Hartmann T, Dohrmann K, Schlee W, Norena A. High-frequency tinnitus without hearing loss does not mean absence of deafferentation. Hear Res 222: 108-114, 2006. doi:10.1016/j.heares. 2006.09.003.

41. Hamani C, Temel Y. Deep brain stimulation for psychiatric disease: contributions and validity of animal models. Sci Trans/ Med 4: 142rv8, 2012. doi:10.1126/scitransImed.3003722.

42. Bauer CA, Turner JG, Caspary DM, Myers KS, Brozoski TJ. Tinnitus and inferior colliculus activity in chinchillas related to three distinct patterns of cochlear trauma. J Neurosci Res 86: 2564-2578, 2008. doi:10.1002/jnr.21699.

43. Dong S, Mulders WH, Rodger J, Robertson D. Changes in neuronal activity and gene expression in guinea-pig auditory brainstem after unilateral partial hearing loss. Neuroscience 159: 1164-1174, 2009. doi:10.1016/j.neuroscience.2009.01.043.

44. Sametsky EA, Turner JG, Larsen D, Ling L, Caspary DM. Enhanced GABAA-mediated tonic inhibition in auditory thalamus of rats with behavioral evidence of tinnitus. J Neurosci 35: 9369-9380, 2015. doi:10.1523/JNEUROSCI.5054-14.2015.

45. Llinas R, Urbano FJ, Leznik E, Ramirez RR, van Marle HJ. Rhythmic and dysrhythmic thalamocortical dynamics: GABA systems and the edge effect. Trends Neurosci 28: 325-333, 2005. doi:10.1016/j.tins. 2005.04.006. 